\title{
Calibration factor comparison between TÜBITAK UME and SASO NMCC
}

\author{
Erkan Danaci ${ }^{1, *}$, Murat Celep $^{1}$, Handan Sakarya ${ }^{1}$, Ozlen Tuncel $^{1}$, Khalid Saleh \\ AlDawood $^{2}$, Ibrahim Mohammed AlBoraih ${ }^{2}$ and Ahmed Hamad AlJawan ${ }^{2}$ \\ ${ }^{1}$ TÜBİTAK Ulusal Metroloji Enstitüsü, Dr. Zeki Acar Cad. No:1, Gebze-Kocaeli, Turkey \\ ${ }^{2}$ SASO National Measurement and Calibration Center, P.O. Box 3437, 11471, Riyadh, Kingdom of \\ Saudi Arabia
}

\begin{abstract}
Bilateral comparison of calibration factor of microwave power sensor was carried out between National Metrology Institute of Turkey (TÜBİTAK UME) and National Measurement and Calibration Center at Saudi Standards, Metrology and Quality Organization of the Kingdom of Saudi Arabia (SASO NMCC). In this comparison, TÜBİTAK UME was pilot laboratory and supplied the travelling standard with $\mathrm{N}$ type connector. Nine different frequency points at $0 \mathrm{dBm}$ power level were determined for this comparison in prepared technical protocol. Each participant used their own measurement standards and applied the direct comparison transfer method at comparison. There is a good agreement between TÜBİTAK UME and SASO NMCC results.
\end{abstract}

\section{Introduction}

Calibration factor (CF) is a parameter used for characterization of microwave power sensors. Power sensors have a fundamental importance for traceability of radio frequency (RF) and microwave power measurements. CF of a power sensor is evaluated by comparative measurements using a power sensor with known parameters $[1,2]$. There are several methods for power sensor calibration used by calibration laboratories and national metrology institutes. One of the most popular method is called direct comparison transfer method and this method produces relatively low uncertainty [3-7].

This paper describes a bilateral comparison on power sensor calibration between National Metrology Institute of Turkey (TÜBİTAK UME) and National Measurement and Calibration Center at Saudi Standards, Metrology and Quality Organization of the Kingdom of Saudi Arabia (SASO NMCC). TÜBITAK UME has a long standing experience in power sensor calibration and SASO NMCC RF \& Microwave Laboratory has recently established a new power sensor calibration setup which is working from $100 \mathrm{kHz}$ to $50 \mathrm{GHz}$ with $\mathrm{N}$ type, 3.5 $\mathrm{mm}$ and $2.4 \mathrm{~mm}$ connectors. The main goal of the comparison is to verify newly installed SASO NMCC infrastructure and to assess the standing of SASO NMCC in RF \& microwave measurement capability.

\footnotetext{
* Corresponding author: erkan.danaci@tubitak.gov.tr
} 


\section{Measured quantity and travelling standard}

$\mathrm{CF}$ of a power sensor has been measured in this comparison and defined as;

$$
C F=\frac{P_{D C}}{P_{I N}}
$$

where; $P_{D C}$ is the DC power measured by power meter and $P_{I N}$ is the RF power incident to power sensor used as standard at selected frequencies.

TÜBITAK UME which is pilot laboratory provided a power sensor as a travelling standard for this comparison. A technical protocol in accordance with the BIPM CCEM guidelines [8] was prepared and the both laboratories measured travelling standard according to this protocol. Beside $\mathrm{CF}$, additionally the comparison covers the reflection coefficient measurement of the travelling standard.

HP 8481A model with type $\mathrm{N}$ connector power sensor was used as the travelling standard due to being in traceability chain and its stability. Both laboratories measured the travelling standard's $\mathrm{CF}$ at the following frequencies at $0 \mathrm{dBm}$ reference power level: 10 $\mathrm{MHz}, 50 \mathrm{MHz}, 500 \mathrm{MHz}, 1 \mathrm{GHz}, 4 \mathrm{GHz}, 8 \mathrm{GHz}, 12 \mathrm{GHz}, 15 \mathrm{GHz}$ and $18 \mathrm{GHz}$. The comparison lasted three months.

According to the protocol, the power sensor was measured at TÜBITTAK UME twice in total, before sending the standard to SASO NMCC and after it was returned to TÜBITAK UME. The participants performed both connector reflection coefficients of travelling standard connector and CF measurements in controlled environmental conditions. For the reflection coefficient measurements, the laboratories used their own vector network analysers. Uncertainties of the $\mathrm{CF}$ measurements were calculated according to the Guide to the Expression of Uncertainty in Measurement (GUM), JCGM 100 [9] for the level of confidence of approximately 95\%. All the measurement results and uncertainty contributions were evaluated by the pilot laboratory. Comparison reference values and associated uncertainties were analysed and calculated using the measurement results of TÜBITAK UME.

\section{Measurement methods}

The direct comparison transfer method has been chosen as the preferred tool in this study since this method generates relatively low uncertainty. Functional diagram of direct comparison method setup is given in Figure 1. Both participants used their standards and measurement procedure during their measurements. Devices used during the measurements are listed in Table 1.

Table 1. Devices used during the measurements.

\begin{tabular}{|l|l|l|}
\hline \multicolumn{1}{|c|}{ TÜBITTAK UME } & \multicolumn{1}{|c|}{ SASO NMCC } \\
\hline Device Category & \multicolumn{1}{|c|}{ Model } & \multicolumn{1}{c|}{ Model } \\
\hline Signal Generator & R\&S SMP04 & Keysight E8257D \\
\hline Power Splitter & Weinschel 1870A & Agilent 11667A \\
\hline MON Power Sensor & HP 8485A & Agilent N8481A \\
\hline MON Powermeter & HP 437B & Agilent N1914A \\
\hline STD Thermistor Mount & Agilent 8478B & Agilent 8478B \\
\hline STD Powermeter+ Voltmeter & HP 432A + Keithley2182 $(2 \mathrm{Ch})$ & Agilent N432A \\
\hline DUT Powermeter & HP 437B & Agilent N1914A \\
\hline
\end{tabular}


Both laboratories used complex reflection coefficient of the standard sensor (STD), travelling standard (device under test, DUT) and the effective reflection coefficient of the power splitter. They carried these values out with their own vector network analysers.

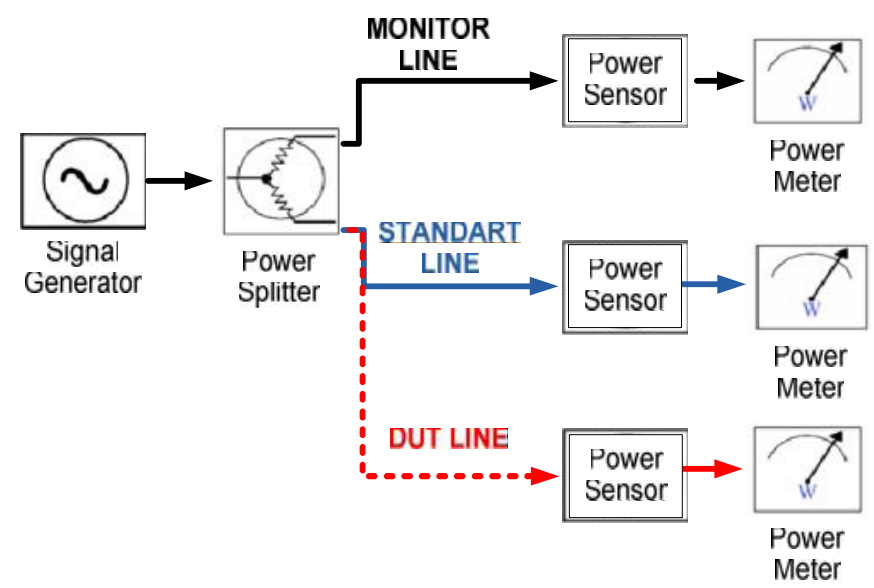

Fig. 1. Function diagram of $\mathrm{CF}$ calibration measurement system according to direct comparison transfer method.

In the direct comparison transfer method, standard power sensor and travelling standard were connected to one of output port of power splitter while monitor power sensor (MON) was attached to the other output port of power splitter. Microwave signal was applied to input port of the power splitter and standard then monitor power was measured. After finishing the measurement, STD was disconnected from power splitter port and DUT was connected immediately to same power splitter port. Then, again microwave power was applied to input port and output powers were measured using DUT and monitor power meters. STD, DUT and related monitor powers were used to calculate calibration factor of the DUT. The mismatch correction was applied to each measurement.

CF of DUT was calculated by Equation 2 in this method.

where;

$$
C F_{D U T}=C F_{S T D} \cdot \frac{P_{D U T}}{P_{S T D}} \cdot \frac{P m_{S} \cdot M_{D}}{P m_{D} \cdot M_{S}}
$$

$C F_{D U T}:$ Calibration factor of DUT,

$C F_{S T D}$ : Calibration factor of STD,

$P_{D U T}:$ Power average of DUT,

$P_{S T D}:$ Power average of STD,

$P m_{D}$ : Measured monitor (MON) power while DUT power sensor is measuring,

$\mathrm{Pm}_{S}$ : Measured MON power while STD power sensor is measuring,

$M_{S} \quad$ : Mismatch between power splitter and STD power sensor,

$M_{D} \quad$ : Mismatch between power splitter and DUT power sensor.

\section{Results}

CF values of travelling standard and comparison reference values $\left(X_{\text {ref }}\right)$ were given in Table 2 for all cycle of the comparison. Comparison reference values and associated uncertainties $\left(U_{r e f}\right)$ were calculated using the measurements result of TÜBİTAK UME as given Equation 3 and Equation 4. 


$$
\begin{aligned}
& X_{\text {ref }}=\frac{U M E 1+U M E 2}{2} \\
& U_{r e f}=\frac{1}{2} \sqrt{U_{U M E 1}^{2}+U_{U M E 2}{ }^{2}}
\end{aligned}
$$

where, $U M E i$ is the $C F_{D U T}$ value of $\mathrm{i}^{\text {th }}$ measurement and $U_{U M E i}$ is uncertainty of $C F_{D U T}$ of $\mathrm{i}^{\text {th }}$ measurement of comparison and $i \square\{1,2\}$.

Degrees of equivalence (DoE) is calculated by subtracting the reference value from the each measurements (in Equation 5) and its uncertainty is calculated according to the Equation 6. DoEs were given in Table 3.

$$
\begin{aligned}
& D o E=X_{l a b}-X_{r e f} \\
& U_{D}=\sqrt{{U_{r e f}}^{2}+U_{l a b}^{2}}
\end{aligned}
$$

The measurement results and associated uncertainties together with the reference values and uncertainties are shown in Figure 2-5.

Table 2. Calibration factor measurement results of HP 8481A power sensor.

\begin{tabular}{|c|c|c|c|c|c|c|c|c|}
\hline Labs & \multicolumn{2}{|c|}{ TÜBİTAK UME1 } & \multicolumn{2}{c|}{ SASO NMCC } & \multicolumn{2}{c|}{ TÜBİTAK UME2 } & \multicolumn{2}{c|}{ Reference Value } \\
\hline Frequencies & $C F_{\text {DUT }}$ & $\begin{array}{c}\text { Unc. } \text { Of } \\
C F_{\text {DUT }}\end{array}$ & $C F_{\text {DUT }}$ & $\begin{array}{c}\text { Unc. } \text { Of } \\
C F_{D U T}\end{array}$ & $C F_{D U T}$ & $\begin{array}{r}\text { Unc. Of } \\
C F_{D U T}\end{array}$ & $X_{\text {ref }}$ & $U_{\text {ref }}$ \\
\hline $10 \mathrm{MHz}$ & 0.9736 & 0.0097 & 0.9869 & 0.0084 & 0.9911 & 0.0099 & 0.9823 & 0.0069 \\
\hline $500 \mathrm{MHz}$ & 0.9904 & 0.0082 & 0.9943 & 0.0084 & 0.9932 & 0.0082 & 0.9918 & 0.0058 \\
\hline $1 \mathrm{GHz}$ & 0.9880 & 0.0083 & 0.9889 & 0.0085 & 0.9880 & 0.0083 & 0.9880 & 0.0059 \\
\hline $4 \mathrm{GHz}$ & 0.9774 & 0.0083 & 0.9802 & 0.0089 & 0.9798 & 0.0082 & 0.9786 & 0.0058 \\
\hline $8 \mathrm{GHz}$ & 0.9636 & 0.0107 & 0.9662 & 0.0132 & 0.9659 & 0.0105 & 0.9647 & 0.0075 \\
\hline $12 \mathrm{GHz}$ & 0.9483 & 0.0095 & 0.9519 & 0.0127 & 0.9523 & 0.0097 & 0.9503 & 0.0068 \\
\hline $15 \mathrm{GHz}$ & 0.9366 & 0.0083 & 0.9426 & 0.0100 & 0.9366 & 0.0087 & 0.9366 & 0.0060 \\
\hline $18 \mathrm{GHz}$ & 0.9388 & 0.0184 & 0.9418 & 0.0242 & 0.9439 & 0.0172 & 0.9413 & 0.0126 \\
\hline
\end{tabular}

Table 3. Degrees of equivalence and its uncertainty.

\begin{tabular}{|c|c|c|c|c|c|c|}
\hline Labs & \multicolumn{2}{|c|}{ TÜBİTAK UME1 } & \multicolumn{2}{c|}{ SASO NMCC } & \multicolumn{2}{c|}{ TÜBİTAK UME2 } \\
\hline Frequencies & $D o E$ & $U_{D}$ & $D o E$ & $U_{D}$ & $D o E$ & $U_{D}$ \\
\hline $10 \mathrm{MHz}$ & -0.0088 & 0.0119 & 0.0046 & 0.0109 & 0.0088 & 0.0121 \\
\hline $500 \mathrm{MHz}$ & -0.0014 & 0.0101 & 0.0025 & 0.0102 & 0.0014 & 0.0101 \\
\hline $1 \mathrm{GHz}$ & 0.0000 & 0.0101 & 0.0009 & 0.0104 & 0.0000 & 0.0102 \\
\hline $4 \mathrm{GHz}$ & -0.0012 & 0.0102 & 0.0016 & 0.0107 & 0.0012 & 0.0101 \\
\hline $8 \mathrm{GHz}$ & -0.0011 & 0.0131 & 0.0014 & 0.0152 & 0.0011 & 0.0129 \\
\hline $12 \mathrm{GHz}$ & -0.0020 & 0.0117 & 0.0016 & 0.0144 & 0.0020 & 0.0118 \\
\hline $15 \mathrm{GHz}$ & 0.0000 & 0.0103 & 0.0060 & 0.0117 & 0.0000 & 0.0106 \\
\hline $18 \mathrm{GHz}$ & -0.0026 & 0.0223 & 0.0005 & 0.0273 & 0.0026 & 0.0213 \\
\hline
\end{tabular}




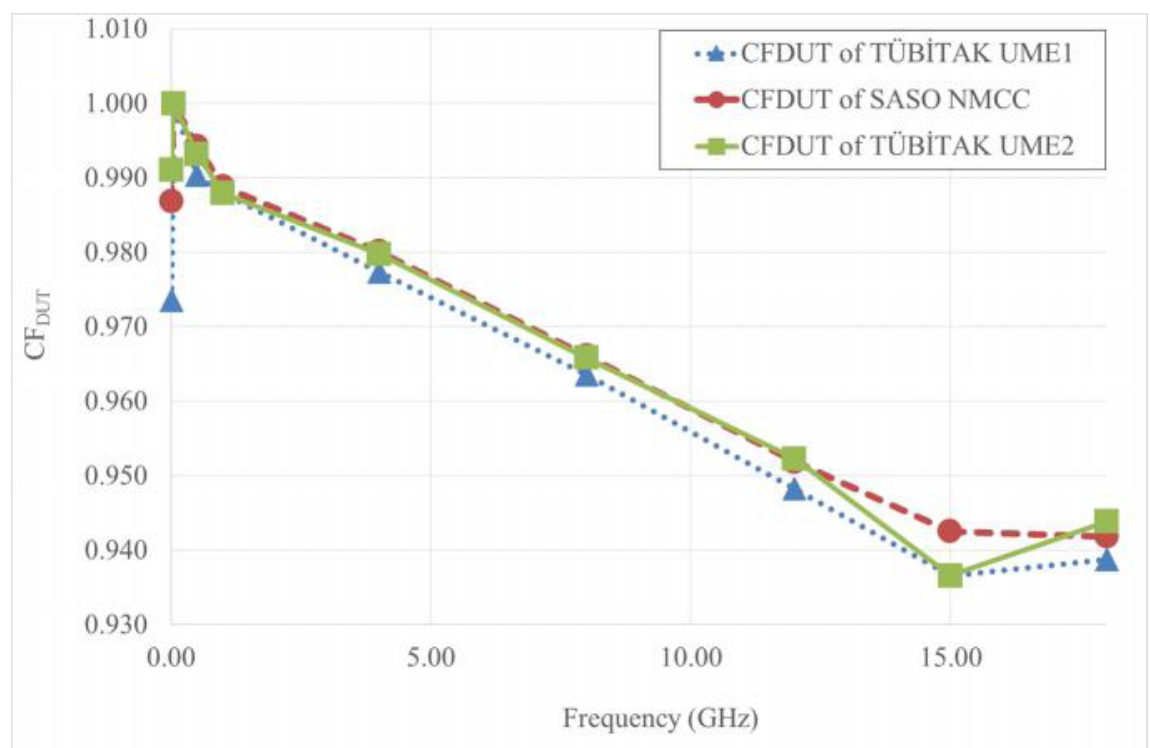

Fig. 2. Calibration factor of the travelling standard of comparison of TÜBİTAK UME and SASO NMCC.

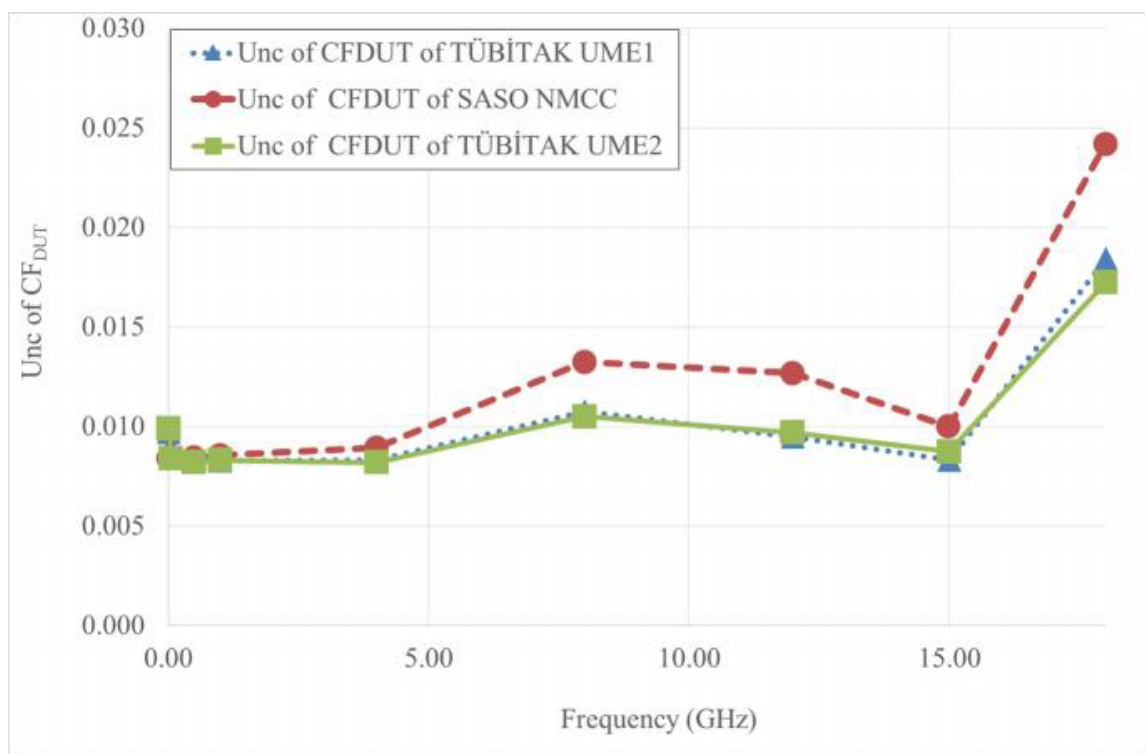

Fig. 3. Uncertainty values of calibration factor of the travelling standard of participants. 


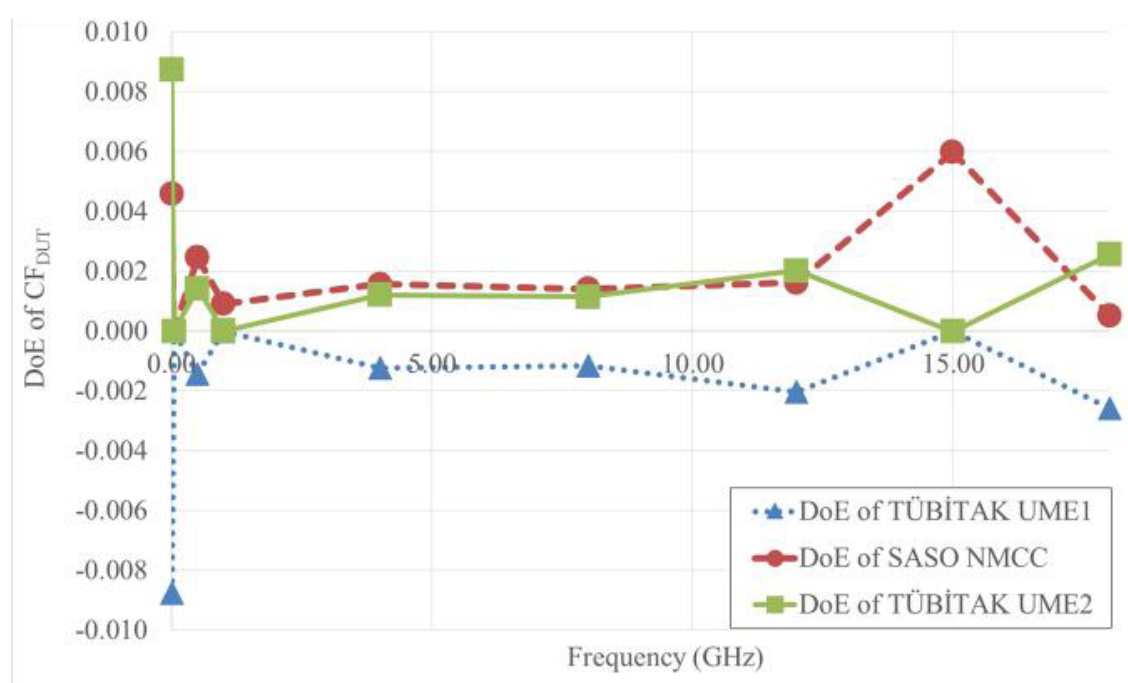

Fig. 4. DoE of calibration factors of the travelling standard.

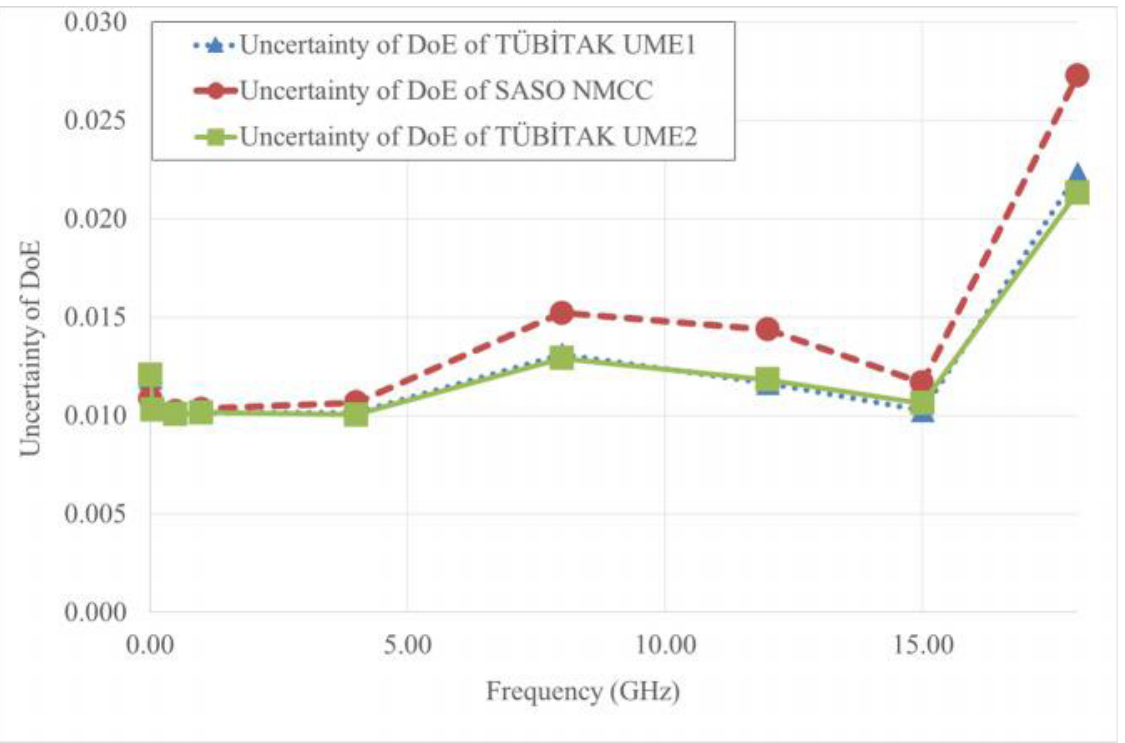

Fig. 5. Uncertainty of DoE.

\section{Conclusion}

Bilateral comparison between TÜBİTAK UME and SASO NMCC was organized. The comparison were completed in three months. Each laboratories measured the traveeling standard using their own measurement setup and procedure. The measurement results show a good agreement between TÜBITTAK UME and SASO NMCC as a conclusion. Degrees of equivalence and uncertainty were calculated. Maximum DoE value is lower than \pm 0.01 and uncertainty is lower than 0.03 . The comparison is regarded as successful at all frequencies. 
This comparison proves the fact that SASO NMCC is a capable metrology institute in RF $\&$ microwave power sensor calibration.

\section{References}

1. A. Famton, "Radio frequency \& microwave power measurement", IEE Electrical Measurement Series 7, Peter Peregrinus Ltd, London, UK (1990)

2. G.R. Orford, "RF power-review of current techniques for power sensor calibration", Accreditation of RF Measurement, IEE Colloquium on, pp:5/1-10 (1993)

3. M.P. Weidman, "Direct Comparison Transfer of Microwave Power Sensor Calibration", NIST Technical Note 1379 (1996)

4. J. R. Juroshek, "NIST 0.05-50 GHz direct-comparison power calibration system", CPEM Conference Proceeding, pp.166-167, Sydney, Australia (2000)

5. K. Yhland, J.R. Judaschke, F. Rausche, C. Wingqvist, J. Stenarson, "Bilateral comparison on calibration factor of a $2.92 \mathrm{~mm}$ power sensor", CPEM Conference Proceeding, pp. 86-87, Maryland, USA (2012)

6. Y. Cai, Y. Shan, Z. Lin, H. Neo, "Coaxial power sensor calibration with adaptor by direct comparison transfer", CPEM Conference Proceeding, pp. 90-91, Maryland, USA (2012)

7. T.M. Wallis, T.P. Crowley, D.X. LeGolvan, R.A. Ginley, "A direct comparison system for power calibration up to $67 \mathrm{GHz}$ ", CPEM Conference Proceeding, pp. 726-727, Maryland, USA (2012)

8. CCEM Guidelines for Planning, Organizing, Conducting and Reporting Key, Supplementary and Pilot Comparisons, (available on the BIPM: http://www.bipm.org/utils/common/pdf/CC/CCEM/ccem_guidelines.pdf) (2007)

9. Evaluation of measurement data - Guide to the Expression of Uncertainty in Measurement (GUM), JCGM 100, First edition, (available on the BIPM : http://www.bipm.org/utils/common/documents/jcgm/JCGM_100_2008_E.pdf) (2008) 